\title{
THE MAYER HOMOLOGY THEORY
}

\author{
EDWIN H. SPANIER ${ }^{1}$
}

1. Introduction. In $1942 \mathrm{~W}$. Mayer [4] ${ }^{2}$ defined new homology groups based on a boundary operator whose $p$ th power ( $p$ a prime) is zero, instead of the usual one whose square is zero. As a coefficient group an abelian group $G$ with only elements of order $p$ is used. The Mayer homology groups $H_{n, q}$ depend on two integers: $n \geqq 0$ and $0<q<p$. Mayer has established the topological invariance of these groups but left unsettled the question of their relation with the classical homology groups. This question is settled in this paper. The answer is embodied in the following theorem which is the main result of the paper.

THEOREM 1.1. Let $p$ be a prime and $G$ an abelian group with all elements of order $p$. The Mayer homology groups $H_{n, q}($ over $G)$ are then related to the classical homology groups $H_{r}$ (over $G$ ) as follows:

(1) If $n \equiv q-1(\bmod p)$, then $H_{n, q} \approx H_{r}$ for $r=2(n-q+1) / p$.

(2) If $n \equiv-1(\bmod p)$, then $H_{n, q} \approx H_{r}$ for $r=2(n+1) / p-1$.

(3) In all other cases, $H_{n, q}=0$.

Conversely, the classical groups $H_{r}$ can be expressed in terms of the Mayer groups as follows:

(4) If $r$ is even, then $H_{r} \approx H_{n, q}$ provided $n-q=p r / 2-1$.

(5) If $r$ is odd, then $H_{r} \approx H_{n, q}$ provided $n=p(r+1) / 2-1$.

The theorem implies that the Mayer groups do not yield new topological invariants but lead instead to rather interesting alternative definitions of the classical homology groups.

The theorem is valid for the absolute and relative homology groups in simplicial complexes. It also remains valid for arbitrary spaces provided the Cech limiting process is used to define both $H_{n, q}$ and $H_{r}$.

The proof of the theorem is not obtained by a direct construction of the requisite isomorphisms but is an application of the axiomatic characterization of homology theory of Eilenberg and Steenrod (sketched in [1] and fully developed in a forthcoming book). Roughly speaking, the procedure is the following. It is shown that certain collections of the Mayer groups, suitably relabeled and together

\footnotetext{
Presented to the Society, September 9, 1948; received by the editors February 28, 1948.

${ }^{1}$ Frank B. Jewett Fellow, 1947-1948.

${ }^{2}$ Numbers in brackets refer to bibliography at the end of the paper.
} 
with suitably defined homomorphisms, satisfy the axioms for homology. The uniqueness theorem in the axiomatic theory then implies that these groups are isomorphic with the classical homology groups based on the same coefficient groups. The theorem follows from a discussion of the coefficient groups and the relabeling process.

The main interest of this paper lies in the fact that it offers an application of the axiomatic approach to a problem which did not seem easy to handle by direct methods. Of course, an analysis of the proof of the uniqueness theorem will yield a direct, though most likely complicated, definition of the isomorphisms.

The next section summarizes all the needed definitions and results from the book being prepared by Eilenberg and Steenrod, to whom the author is indebted for allowing him access to the manuscript.

2. The axioms. Elienberg and Steenrod [1] have characterized homology theory on a certain class of spaces by means of a set of axioms which are topological in nature. In addition, another set (to appear in their forthcoming book) is considered which is combinatorial in nature and characterizes homology theory for simplicial complexes. This latter set is the one we employ in the following.

DEFINITION 2.1. Two simplicial maps $f$ and $g$ of a simplicial pair $(K, L)$ (consisting of a simplicial complex $K$ and a closed subcomplex $L$ ) into a simplicial pair $\left(K^{\prime}, L^{\prime}\right)$ are said to be contiguous if for every simplex $s$ of $K$ (or $L$ ) the simplexes $f(s)$ and $g(s)$ are contained in a simplex of $K^{\prime}$ (or $L^{\prime}$ ).

Definition 2.2. A sequence of groups and homomorphisms

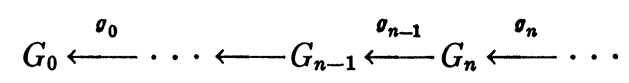

is said to be exact if for $n \geqq 1$ the kernel of $g_{n-1}$ is the image of $g_{n}$, and $g_{0}$ is a homomorphism onto.

Definition 2.3. Consider a system $H=\left\{H_{r}(K, L), \partial, f_{*}\right\}$ consisting of the following concepts:

(a) For every simplicial pair $(K, L)$ and integer $r \geqq 0, H_{r}(K, L)$ is an abelian group called the $n$th homology group of $K \bmod L$.

(b) For $r \geqq 1, \partial: H_{r}(K, L) \rightarrow H_{r-1}(L)$ is a homomorphism called the boundary operator.

(c) For every simplicial map $f:(K, L) \rightarrow\left(K^{\prime}, L^{\prime}\right)$ there is a homomorphism $f_{*}: H_{r}(K, L) \rightarrow H_{r}\left(K^{\prime}, L^{\prime}\right)$ called the homomorphism induced by $f$.

The system $H$ is called a simplicial homology theory if it satisfies the following axioms: 
Aхгом 1. If $f$ is the identity simplicial map of $(K, L)$ onto itself, then $f_{*}$ is the identity isomorphism of $H_{r}(K, L)$ onto itself.

AxIom 2. If $f:(K, L) \rightarrow\left(K^{\prime}, L^{\prime}\right)$ and $g:\left(K^{\prime}, L^{\prime}\right) \rightarrow\left(K^{\prime \prime}, L^{\prime \prime}\right)$ are simplicial, then $(g f)_{*}=g_{*} f_{*}$.

AхIом 3. If $f:(K, L) \rightarrow\left(K^{\prime}, L^{\prime}\right)$ is simplicial and $n \geqq 1$, then commutativity holds in the diagram

$$
\begin{array}{cc}
H_{r}(K, L) \stackrel{f_{*}}{\longrightarrow} & H_{r}\left(K^{\prime}, L^{\prime}\right) \\
\partial \downarrow & \downarrow \partial \\
H_{r-1}(L) \stackrel{(f \mid L)_{*}}{\longrightarrow} & H_{r-1}\left(L^{\prime}\right) .
\end{array}
$$

Axiom 4 (Exactness axiom). If $i: L \rightarrow K$ and $j: K \rightarrow(K, L)$ denote identity simplicial maps, then the following sequence is exact $H_{0}(K, L) \stackrel{j_{*}}{\longleftarrow} . \stackrel{i_{*}}{\longleftarrow} H_{r}(K) \stackrel{i_{*}}{\longleftarrow} H_{r}(L) \stackrel{\text { ə }}{\longleftarrow} H_{r+1}(K, L) \stackrel{j_{*}}{\longleftarrow} \ldots$.

Axıом 5 (Contiguity axiom). If $f, g:(K, L) \rightarrow\left(K^{\prime}, L^{\prime}\right)$ are contiguous simplicial maps, then $f_{*}=g_{*}$.

Aхгом 6 (Excision axiom). If $K_{1}, K_{2}$ are closed subcomplexes of a complex $K$, the homomorphisms

$$
i_{*}: \quad H_{r}\left(K_{1}, K_{1} \cap K_{2}\right) \rightarrow H_{r}\left(K_{1} \cup K_{2}, K_{2}\right)
$$

induced by the identity map $i:\left(K_{1}, K_{1} \cap K_{2}\right) \rightarrow\left(K_{1} \cup K_{2}, K_{2}\right)$ are isomorphisms onto.

Aхгом 7 (Dimension axiom). If $P$ is a complex consisting of a single vertex, then $H_{r}(P)=0$ for $r>0$.

If a simplicial homology theory is given, the group $H_{0}(P)$ is called the coefficient group of the theory. If $G$ is a fixed abelian group, the groups $H_{r}(K, L)=H_{r}(K, L ; G)$, defined in terms of the cellular structure of $K$ as in [3, chap. 3] together with suitable definitions of $\partial$ and $f_{*}$ form a simplicial homology theory with $G$ as coefficient group. This homology theory will be referred to as the classical homology theory.

The above shows that simplicial homology theories with arbitrary coefficient group exist. The following uniqueness theorem, which will appear in the book on the axiomatic theory mentioned earlier, shows that any simplicial homology theory is isomorphic to the classical homology theory with a suitable coefficient group.

TheOREM 2.4. Given two simplicial homology theories $H$ and $\bar{H}$ and given an isomorphism 


$$
h_{0}: G \approx \bar{G}
$$

of their coefficient groups, there exists an isomorphism

$$
h: H \approx \bar{H}
$$

which is an extension of $h_{0}$.

By an isomorphism $h: H \approx \bar{H}$ between two simplicial homology theories is meant an isomorphism between corresponding homology groups $H_{r}(K, L)$ and $\bar{H}_{r}(K, L)$ which commutes with the boundary operator and induced homomorphism.

3. Definition of the Mayer groups. Let $p$ be a fixed prime and let $G$ be a fixed abelian group all of whose elements are of order $p$.

Definition 3.1. Let $K$ be a finite simplicial complex with vertices $\left\{v_{i}\right\}$. An $n$-cell $\sigma^{n}$ of $K$ is defined to be an $(n+1)$-tuple $v_{0}, \cdots, v_{n}$ of vertices $v_{i}$ (not necessarily distinct) such that the vertices $v_{0}, \cdots, v_{n}$ all belong to some simplex of $K$. Two such $(n+1)$-tuples differing only in the arrangement of the $v$ 's are to be considered the same $n$-cell. There are no cells of dimension $k$ for $k<0$.

Definition 3.2. For $n \geqq 0$ the group $C_{n}(K)$ of $n$-chains of $K$ with coefficients in $G$ is the group of linear forms $\sum_{i} g_{i} \sigma_{i}^{n}$ in the $n$-cells $\sigma_{i}^{n}$ of $K$ with coefficients $g_{i} \in G$. An elementary $n$-chain is a chain of the form $g \sigma_{\imath}^{n}$. Clearly the elementary $n$-chains span $C_{n}(K)$.

For $n \geqq 1$, the boundary homomorphism

$$
F: \quad C_{n}(K) \rightarrow C_{n-1}(K)
$$

is defined as follows. It suffices to define $F$ for elementary $n$-chains and extend to $C_{n}(K)$ by linearity. Define $F\left(g\left(v_{0} \cdots v_{n}\right)\right)$ by

$$
F\left(g\left(v_{0} \cdots v_{n}\right)\right)=\sum_{i} g\left(v_{0} \cdots \tilde{v}_{i} \cdots v_{n}\right)
$$

where $\left(v_{0} \cdots \tilde{v}_{i} \cdots v_{n}\right)$ denotes the $(n-1)$-cell obtained from the cell $v_{0} \cdots v_{n}$ by deleting $v_{i}$. The fact that every element of $G$ has order $p$ implies, as shown by Mayer $\left[4\right.$, p. 371], that for $n \geqq p, F^{p}$ maps $C_{n}(K)$ into the zero of $C_{n-p}(K)$.

Definition 3.3. Let $q$ be an integer such that $0<q<p$. If $n \geqq q$, consider the diagram

$$
C_{n+p-q}(K) \stackrel{F^{p-q}}{\longrightarrow} C_{n}(K) \stackrel{F^{q}}{\longrightarrow} C_{n-q}(K) .
$$

The group $Z_{n, q}(K)$ of $q$ th $n$-dimensional cycles of $K$ is defined to be the kernel of $F^{q}$, and the group $B_{n, q}(K)$ of $q$ th $n$-dimensional boundaries of $K$ is defined to be the image of $F^{p-q}$. If $0 \leqq n<q$, we define 
$Z_{n, q}(K)$, to be $C_{n}(K)$ and $B_{n, q}(K)$ to be the image of $C_{n+p-q}(K)$ under $F^{p-q}$. Since $F^{p}=0$, it follows that $B_{n, q}(K) \subset Z_{n, q}(K)$. The factor group

$$
H_{n, q}(K)=Z_{n, q}(K) / B_{n, q}(K)
$$

is defined to be the $q$ th $n$-dimensional homology group of $K$. (This notation differs slightly from that used by Mayer in that he uses $H_{a}^{n}$ to denote the group $H_{n, q}$.

Theorem 3.4. If $P$ is a complex consisting of a single vertex $v$, then

$$
H_{q-1, q}(P) \approx G \text {, and } H_{n, q}(P)=0 \text { for } n \neq q-1 \text {. }
$$

Proof. If $n \geqq 0$, there is only one $n$-cell $\sigma^{n}$ of $P$. Hence, $C_{n}(P)$ consists solely of the elementary chains $g \sigma^{n}$ and is isomorphic to $G$ under the mapping $g \sigma^{n} \rightarrow g$. If $n>0$,

$$
F\left(g \sigma^{n}\right)=((n+1) g) \sigma^{n-1} .
$$

Since $p$ is a prime, elements of $G$ can be divided by integers $m$ if $m \neq 0(\bmod p)$. It follows that if $n+1 \neq \equiv 0(\bmod p), F\left((1 /(n+1)) g \sigma^{n}\right)$ $=g \sigma^{n-1}$. Therefore, for $n>0$,

$$
\begin{array}{lll}
F\left(C_{n}(P)\right)=0 & \text { if } & n \equiv-1(\bmod p), \\
F\left(C_{n}(P)\right)=C_{n-1}(P) & \text { if } & n \neq-1(\bmod p) .
\end{array}
$$

It follows that for $n \geqq q$

$$
\begin{aligned}
& F^{q}\left(C_{n}(P)\right)=0 \quad \text { if } \quad n \equiv k \text { with }-1 \leqq k<q-1, \\
& F^{q}\left(C_{n}(P)\right)=C_{n-q}(P) \quad \text { otherwise. }
\end{aligned}
$$

Therefore,

$$
\begin{aligned}
Z_{n, q}(P) & = \begin{cases}C_{n}(P) & \text { if } n=q-1 \text { or } n \equiv k \text { with }-1 \leqq k<q-1, \\
0 & \text { otherwise, }\end{cases} \\
B_{n, q}(P) & = \begin{cases}0 & \text { if } n+p-q \equiv m \text { with }-1 \leqq m<p-q-1, \\
C_{n}(P) & \text { if } n+p-q \equiv m \text { with } p-q-1 \leqq m<p-1 .\end{cases}
\end{aligned}
$$

Then $H_{n, q}(P)=0$ unless $n=q-1$ or $n \equiv k$ with $-1 \leqq k<q-1$. If $n \equiv k$ with $-1 \leqq k<q-1$, then $n+p-q \equiv k+p-q$ and $p-q-1 \leqq k$ $+p-q<p-1$, so $B_{n, q}(P)=C_{n}(P)$, and $H_{n, q}(P)=0$. Therefore, $H_{n, q}(P)=0$ if $n \neq q-1$. If $n=q-1$, then $n+p-q=p-1 \equiv-1$, so $B_{q-1, q}(P)=0$. Since $Z_{q-1, q}(P)=C_{q-1}(P)$, it follows that $H_{q-1, q}(P)$ $=C_{q-1}(P) \approx G$.

4. Relative theory. The axioms stated in $\$ 2$ require that a group $H_{n}(K, L)$ be defined for every simplicial pair $(K, L)$. In this section 
we extend the Mayer definition to the relative case so that later we will have the groups needed in verifying the axioms.

Let $L$ be a (closed) subcomplex of $K$. Then any cell of $L$ is also a cell of $K$ so that $C_{n}(L) \subset C_{n}(K)$. It is clear that $F$ defined for chains of $L$ is the same homomorphism as $F$ defined for chains of $K$ when restricted to $C_{n}(L)$.

Definition 4.1. For $n \geqq q$, the group $Z_{n, q}(K, L)$ of $q$ th $n$-dimensional cycles of $K \bmod L$ is defined to be the subgroup of $C_{n}(K)$ which is the inverse image of $C_{n-q}(L)$ under $F^{q}$, while for $n<q, Z_{n, q}(K, L)$ is defined to be $C_{n}(K)$. The group $B_{n, q}(K, L)$ of $q$ th $n$-dimensional boundaries of $K \bmod L$ is defined to be the subgroup of $C_{n}(K)$ spanned by the two subgroups $C_{n}(L)$ and $F^{p-q}\left(C_{n+p-q}(K)\right)$. Then $B_{n, q}(K, L)$ $\subset Z_{n, q}(K, L)$ and the factor group

$$
H_{n, q}(K, L)=Z_{n, q}(K, L) / B_{n, q}(K, L)
$$

is defined to be the $q$ th $n$-dimensional homology group of $K \bmod L$.

Note. If $L=0$ is the null set, then $H_{n, q}(K, 0)=H_{n, q}(K)$.

In the following we use $\phi: Z_{n, q}(K, L) \rightarrow H_{n, q}(K, L)$ to denote the natural homomorphism of $Z_{n, q}(K, L)$ onto its factor group $H_{n, q}(K, L)$.

Definition 4.2. For $n \geqq q$, define a homomorphism

$$
F^{q}: H_{n, q}(K, L) \rightarrow H_{n-q, p-q}(L)
$$

as follows. Let $z \in Z_{n, q}(K, L)$. Then from the definition of $Z_{n, q}(K, L)$ it follows that $F^{q} z \in C_{n-q}(L)$. If $n-q \geqq p-q$, then $F^{p-q}\left(F^{q} z\right)=F^{p} z=0$. If $n-q<p-q, C_{n-q}(L)=Z_{n-q, p-q}(L)$. In any event we see that $F^{q} z \in Z_{n-q, p-q}(L)$ so that $F^{q}$ is a homomorphism

$$
F^{q}: Z_{n, q}(K, L) \rightarrow Z_{n-q, p-q}(L) .
$$

Let $b \in B_{n, q}(K, L)$. Then there is $u \in C_{n+p-q}(K)$ and $v \in C_{n}(L)$ such that

$$
F^{p-q} u+v=b .
$$

Since $F^{q} b=F^{p} u+F^{q_{v}}=F^{q_{v}} \in B_{n-q, p-q}(L)$, it follows that $F^{q}$ maps $B_{n, q}(K, L)$ into $B_{n-q, p-q}(L)$ and so induces a homomorphism

$$
F^{q}: H_{n, q}(K, L) \rightarrow H_{n-q, p-q}(L)
$$

such that $F^{q} \phi z=\phi F^{q} z$ for $z \in Z_{n, q}(K, L)$.

Definition 4.3. Let $f:(K, L) \rightarrow\left(K^{\prime}, L^{\prime}\right)$ be a simplicial map. If $v_{0}, \cdots, v_{n}$ is an $n$-cell of $K$ (or $\left.L\right), f\left(v_{0}, \cdots, v_{n}\right)=f\left(v_{0}\right), \cdots, f\left(v_{n}\right)$ is clearly an $n$-cell of $K^{\prime}$ (or $L^{\prime}$ ). Therefore, $f$ induces a homomorphism $f_{n}: C_{n}(K) \rightarrow C_{n}\left(K^{\prime}\right)$ such that $f_{n}\left(\sum_{i} g_{i} \sigma_{i}^{n}\right)=\sum_{i} g_{i} f\left(\sigma_{i}^{n}\right)$. 
LEMMA 4.4. For $n \geqq 1$, commutativity holds in the diagram

$$
\begin{aligned}
C_{n}(K) \stackrel{F}{\longrightarrow} & C_{n-1}(K) \\
f_{n} \downarrow & \stackrel{F}{\longrightarrow} \stackrel{\downarrow f_{n-1}}{\longrightarrow} C_{n-1}\left(K^{\prime}\right) .
\end{aligned}
$$

Proof. It suffices to prove the commutativity for elementary $n$ chains. Let $v_{0} \cdots v_{n}$ be an $n$-cell of $K$. Then

$$
\begin{aligned}
F f_{n}\left(g v_{0} \cdots v_{n}\right) & =F g f\left(v_{0}\right) \cdots f\left(v_{n}\right) \\
& =\sum_{i} g f\left(v_{0}\right) \cdots \tilde{f}\left(v_{i}\right) \cdots f\left(v_{n}\right)
\end{aligned}
$$

and

$$
\begin{aligned}
f_{n-1} F\left(g v_{0} \cdots v_{n}\right) & =f_{n-1}\left(\sum_{i} g v_{0} \cdots \tilde{v}_{i} \cdots v_{n}\right) \\
& =\sum_{i} g f\left(v_{0}\right) \cdots \tilde{f}\left(v_{i}\right) \cdots f\left(v_{n}\right) .
\end{aligned}
$$

It follows from Lemma 4.4 that $f_{n}$ induces a homomorphism

$$
f_{*}: H_{n, q}(K, L) \rightarrow H_{n, q}\left(K^{\prime}, L^{\prime}\right)
$$

such that $f_{* \phi z}=\phi f_{n} z$ for $z \in Z_{n, q}(K, L)$.

Theorem 4.5. If $f:(K, L) \rightarrow(K, L)$ is the identity simplicial map, then $f_{*}$ is the identity isomorphism.

Proof. The theorem follows immediately from the fact that $f_{n}$ is the identity isomorphism of $C_{n}(K)$ onto itself.

Theorem 4.6. If $f:(K, L) \rightarrow\left(K^{\prime}, L^{\prime}\right)$ and $g:\left(K^{\prime}, L^{\prime}\right) \rightarrow\left(K^{\prime \prime}, L^{\prime \prime}\right)$ are simplicial, then $(g f)_{*}=g_{*} f_{*}$.

Proof. $g f$ is simplicial because both $f$ and $g$ are, and $(g f)_{n}=g_{n} f_{n}$ which implies the theorem.

Theorem 4.7. If $f:(K, L) \rightarrow\left(K^{\prime}, L^{\prime}\right)$ is simplicial and if $n \geqq q$, commutativity holds in the diagram

$$
\begin{aligned}
& H_{n, q}(K, L) \stackrel{f_{*}}{\longrightarrow} H_{n, q}\left(K^{\prime}, L^{\prime}\right) \\
& \quad F^{q} \downarrow \\
& H_{n-q, p-q}(L) \stackrel{(f \mid L)_{*}}{\longrightarrow} \underset{F^{q}}{\longrightarrow} H_{n-q, p-q}\left(L^{\prime}\right) .
\end{aligned}
$$

Proof. It follows from Lemma 4.4 that 


$$
F^{q} f_{n}=(f \mid L)_{n-q} F^{q}
$$

and this, together with the commutativity of $F^{q}$ and $\phi$, implies the theorem.

Theorem 4.8. If $f, g:(K, L) \rightarrow\left(K^{\prime}, L^{\prime}\right)$ are contiguous, then $f_{*}=g_{*}$.

Proof. This is proved for the absolute case by Mayer [4, p. 379]. The same proof carries over to the relative case without any essential change and so is omitted here.

Theorem 4.9. If $K_{1}, K_{2}$ are closed subcomplexes of $K$, and if $i:\left(K_{1}, K_{1} \cap K_{2}\right) \rightarrow\left(K_{1} \cup K_{2}, K_{2}\right)$ is the identity simplicial map, then

$$
i_{*}: H_{n, q}\left(K_{1}, K_{1} \cap K_{2}\right) \approx H_{n, q}\left(K_{1} \cup K_{2}, K_{2}\right) .
$$

PROOF. Since $C_{n}\left(K_{1} \cap K_{2}\right)=C_{n}\left(K_{1}\right) \cap C_{n}\left(K_{2}\right)$ and $C_{n}\left(K_{1} \cup K_{2}\right)$ $=C_{n}\left(K_{1}\right)+C_{n}\left(K_{2}\right)=$ the subgroup of $C_{n}(K)$ spanned by $C_{n}\left(K_{1}\right)$ and $C_{n}\left(K_{2}\right)$, it follows from the Noëther isomorphism theorem that

$$
C_{n}\left(K_{1}\right) / C_{n}\left(K_{1} \cap K_{2}\right) \approx C_{n}\left(K_{1} \cup K_{2}\right) / C_{n}\left(K_{2}\right) .
$$

More precisely, if $\tau: C_{n}\left(K_{1} \cup K_{2}\right) \rightarrow C_{n}\left(K_{1} \cup K_{2}\right) / C_{n}\left(K_{2}\right)$ is the natural homomorphism, then $\tau i_{n}$ maps $C_{n}\left(K_{1}\right)$ onto $C_{n}\left(K_{1} \cup K_{2}\right) / C_{n}\left(K_{2}\right)$ and has kernel $C_{n}\left(K_{1} \cap K_{2}\right)$.

If $n \geqq q$ and $z \in Z_{n, q}\left(K_{1} \cup K_{2}, K_{2}\right)$, then $z \in C_{n}\left(K_{1} \cup K_{2}\right)$ such that $F^{q} z \in C_{n-q}\left(K_{2}\right)$. Choose $z^{\prime} \in C_{n}\left(K_{1}\right)$ such that $\tau i_{n} z^{\prime}=\tau z$. Then $i_{n} z^{\prime}$ $=z+c$ for some $c \in C_{n}\left(K_{2}\right)$ and so

$$
\tau F^{q} i_{n} z^{\prime}=\tau F^{q}(z+c)=\tau\left(F^{q} z+F^{a} c\right)=0
$$

because $F^{q} z$ and $F^{q} c$ both belong to $C_{n-q}\left(K_{2}\right)$. Therefore, $\tau i_{n-q} F^{q_{z^{\prime}}}$ $=\tau F^{a} i_{n} z^{\prime}=0$, so $F^{q} z^{\prime}$ is an $(n-q)$-chain of $K_{1} \cap K_{2}$ from which it follows that $z^{\prime}$ is a $q$ th $n$-cycle of $K_{1} \bmod K_{1} \cap K_{2}$. Then we have $\phi z^{\prime} \in H_{n, q}\left(K_{1}, K_{1} \cap K_{2}\right)$ and

$$
i_{*} \phi z^{\prime}=\phi i_{n} z^{\prime}=\phi(z+c)=\phi z
$$

the last equality because $c \in B_{n, q}\left(K_{1} \cup K_{2}, K_{2}\right)$. Therefore, $i_{*}$ maps $H_{n, q}\left(K_{1}, K_{1} \cap K_{2}\right)$ onto $H_{n, q}\left(K_{1} \cup K_{2}, K_{2}\right)$ if $n \geqq q$.

If $n<q$, the same proof as above can be used, the only difference being that it is no longer necessary to show that $z^{\prime}$ is a $q$ th $n$-cycle of $K_{1} \bmod K_{1} \cap K_{2}$ because any $n$-chain of $K_{1}$ is such a cycle by definition. Hence, $i_{*}$ is onto in all cases.

To prove the kernel of $i_{*}$ is zero let $i_{*} \phi z=0$. Then $i_{n} z=F^{p-q} C+d$ where $c \in C_{n+p-q}\left(K_{1} \cup K_{2}\right)$ and $d \in C_{n}\left(K_{2}\right)$. Choose $c^{\prime} \in C_{n+p-q}\left(K_{1}\right)$ such that $\tau i_{n+p-q} c^{\prime}=\tau c$. Then $c-i_{n+p-q} c^{\prime} \in C_{n+p-q}\left(K_{2}\right)$. Now $z-F^{p-q} C^{\prime}$ $\in Z_{n, q}\left(K_{1}, K_{1} \cap K_{2}\right)$ and 


$$
\tau i_{n}\left(z-F^{p-q} c^{\prime}\right)=\tau F^{p-q} c+\tau d-\tau F^{p-q} i_{n+p-q} c^{\prime}
$$

which equals zero because $F^{p-q}\left(c-i_{n+p-q} c^{\prime}\right)$ and $d$ both belong to $C_{n}\left(K_{2}\right)$. Since the kernel of $\tau i_{n}$ is $C_{n}\left(K_{1} \cap K_{2}\right)$, we see that $z-F^{p-q} c^{\prime}$ $\in C_{n}\left(K_{1} \cap K_{2}\right)$. Hence $z=F^{p-q} c^{\prime}+d^{\prime}$ for some $d^{\prime} \in C_{n}\left(K_{1} \cap K_{2}\right)$. It follows that $\phi z=0$, so $i_{*}$ is an isomorphism.

5. Verification of the axioms. All of the concepts necessary to verify the axioms have been defined, and the main theorem can now be proved.

A pair of integers $(q, k)$ is called admissible if $0<q<p$ and $0 \leqq k<q$. For a fixed admissible pair $(q, k)$ we define a simplicial homology theory $H^{q, k}=\left\{H_{r}^{q, \boldsymbol{k}}, \partial, f_{*}\right\}$ as follows.

Define

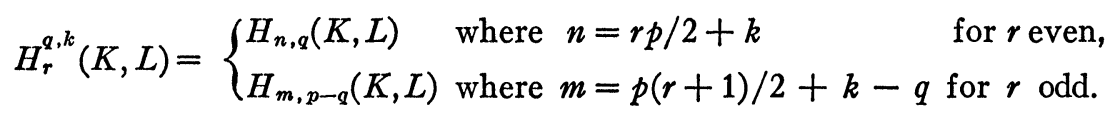
The boundary operator $\partial: H_{r}^{q, \mathbf{k}}(K, L) \rightarrow H_{r-1}^{q, \mathbf{k}}(L)$ is defined to be the homomorphism

$$
\begin{aligned}
& F^{q}: H_{n, q}(K, L) \rightarrow H_{n-q, p-q}(L) \quad \text { if } r \text { is even, } \\
& F^{p-q}: H_{m, p-q}(K, L) \rightarrow H_{m-p+q, q}(L) \quad \text { if } r \text { is odd. }
\end{aligned}
$$

The homomorphism

$$
f_{*}: H_{r}^{q, k}(K, L) \rightarrow H_{r}^{q, k}\left(K^{\prime}, L^{\prime}\right)
$$

induced by a simplicial map $f:(K, L) \rightarrow\left(K^{\prime}, L^{\prime}\right)$ is defined to be the homomorphism

$$
\begin{aligned}
f_{*}: H_{n, q}(K, L) & \rightarrow H_{n, q}\left(K^{\prime}, L^{\prime}\right) \\
f_{*}: H_{m, p-q}(K, L) & \rightarrow H_{m, p-q}\left(K^{\prime}, L^{\prime}\right) \quad \text { if } r \text { is even, }
\end{aligned}
$$

It will be shown that $\left\{H_{r}^{q, \boldsymbol{t}}, \partial, f_{*}\right\}$ is a simplicial homology theory. Theorems 4.5-4.9 and 3.4 imply all the axioms except for Axiom 4, the exactness axiom. To prove exactness, consider the following sequence

$$
\ldots \stackrel{F_{p-q}}{\longrightarrow} C_{r p / 2+k}(K) \stackrel{F_{q}}{\longrightarrow} C_{r p / 2+k-q}(K) \stackrel{F_{p-q}}{\longrightarrow} \ldots \stackrel{F_{p-q}}{\longrightarrow} C_{k}(K)
$$

and subsequence

$$
\ldots \stackrel{p_{p-q}}{\longrightarrow} C_{r p / 2+k}(L) \stackrel{F_{q}}{\longrightarrow} C_{r p / 2+k-q}(L) \quad \stackrel{p_{p-q}}{\longrightarrow} \ldots \stackrel{p_{p-q}}{\longrightarrow} C_{k}(L) .
$$

The groups $H_{r}^{q, \boldsymbol{t}}(K, L)$ have been defined to be the homology groups 
of the upper sequence modulo the lower sequence (in the notation of Kelley-Pitcher [2]). It follows that the homology sequence of $(K, L)$ is exact [2, Theorem 3.3].

We have now shown that for any admissable pair $(q, k)$ there is a corresponding homology theory. The uniqueness theorem stated in $\$ 2$ implies that this homology theory is the same as the classical homology theory based on $H_{0}^{q, k}(P)$ as coefficient group. By Theorem 3.4,

$$
H_{0}^{q, k}(P)=H_{k, q}(P)= \begin{cases}G & \text { if } k=q-1, \\ 0 & \text { if } k \neq q-1 .\end{cases}
$$

Hence, for $k \neq q-1$, the homology theory $H^{q, k}$ is trivial because it is isomorphic to the classical one based on a trivial coefficient group. For $k=q-1$, the homology theory $H^{q, q-1}$ is isomorphic to the classical one based on $G$ as coefficient group. Hence, if $H_{r}(K, L)$ denotes the classical homology group of $K \bmod L$ based on $G$ as coefficient group, we see that

$$
H_{r}(K, L)=\left\{\begin{array}{r}
H_{r}^{q, q-1}(K, L)=H_{n, q}(K, L) \\
\quad \text { where } n=p r / 2+q-1 \text { for } r \text { even, } \\
H_{r}^{p-q, p-q-1}(K, L)=H_{m, q}(K, L) \\
\text { where } m=p(r+1) / 2-1 \text { for } r \text { odd, }
\end{array}\right.
$$

thus parts (4) and (5) of Theorem 1.1 have been proved.

Let $H_{n, q}(K, L)$ be any Mayer group. We shall determine this group in terms of the classical groups by showing that it belongs to some homology theory $H^{m, k}$. Find integers $m, t$ such that

$$
n=m p+t \quad \text { where } 0 \leqq t<p .
$$

If $t<q,(q, t)$ is an admissible pair, and

$$
H_{2 m}^{q, t}(K, L)=H_{m p+t, q}(K, L)=H_{n, q}(K, L) .
$$

Hence, if $n \equiv t(\bmod p)$ with $0 \leqq t<q$,

$$
\begin{aligned}
H_{n, q}(K, L) & =H_{2(n-t) / p}^{q, t}(K, L) \\
& =\left\{\begin{array}{lr}
0 & \text { if } t \neq q-1, \\
H_{r}(K, L) & \text { for } r=2(n-q+1) / p \text { if } t=q-1 .
\end{array}\right.
\end{aligned}
$$

This proves (1) of Theorem 1.1.

If $t \geqq q$, then $0 \leqq t-q<p-q$ so $(p-q, t-q)$ is an admissible pair, 
and

$$
H_{2 m+1}^{p-q, t-q}(K, L)=H_{m p+p+t-p, q}(K, L)=H_{n, q}(K, L) .
$$

Hence, if $n \equiv t(\bmod p)$ with $q \leqq t<p$

$$
\begin{aligned}
& H_{n, q}(K, L)=H_{2(n-t) / p+1}^{p-q, t-q}(K, L) \\
& =\left\{\begin{array}{lr}
0 & \text { if } t \neq p-1, \\
H_{r}(K, L) & \text { where } r=2(n+1) / p-1 \text { if } t=p-1,
\end{array}\right.
\end{aligned}
$$

thus proving (2) and (3) of Theorem 1.1.

\section{BIBLIOGRAPHY}

1. S. Eilenberg and N. E. Steenrod, Axiomatic approach to homology theory, Proc. Nat. Acad. Sci. U.S.A. vol. 31 (1945) pp. 117-120.

2. J. L. Kelley and A. E. Pitcher, Exact homomorphism sequences in homology theory, Ann. of Math. vol. 48 (1947) pp. 682-709.

3. S. Lefschetz, Algebraic topology, Amer. Math. Soc. Colloquium Publications, vol. 27, New York, 1942.

4. W. Mayer, A new homology theory, I, II, Ann. of Math. vol. 43 (1942) pp. 370$380,594-605$.

INSTITUTE FOR ADVANCED STUDY 